\title{
A Coaxial-to-CPW Transition for Microwave Breast Cancer Detection Antennas
}

\author{
A. F. Desouky ${ }^{1}$, T.G.Abouelnaga ${ }^{2}$, M.B. Tayel ${ }^{3}$ \\ ${ }^{I}$ (T. A., Communication and Electronics Engineering, HIET/Kafr El-Shiekh, Egypt) \\ ${ }^{2}$ (Researcher, Microstrip Circuits, Electronic Research Institute /Giza, Egypt) \\ ${ }_{2}^{2}$ (Assistant professor Communication and Electronics Engineering, HIET/ Kafr El-Shiekh, Egypt) \\ ${ }_{3}^{3}$ (Professor, Electrical Engineering/ Alexandria University, Egypt)
}

\begin{abstract}
A transition from coaxial-to- coplanar waveguide (CPW) for microwave breast cancer detection antennas is presented and design rules based on analytical calculations and simulated one using CST Microwave Studio 2014 simulator are presented. The measured results show that an operating frequency range extends from $1 \mathrm{GHz}$ to $15 \mathrm{GHz}$ is obtained. This paper discuss mathematical method of coplanar waveguide $(C P W)$ characteristic impedance calculation and also, developed a graphical charts for coplanar waveguide $(C P W)$ design. Both measured and calculated results are compared and good agreement is obtained.
\end{abstract}

Keywords: Coplanar waveguide, Microwave, Breast cancer.

\section{Introduction}

A transition from coaxial-to-coplanar waveguide (CPW) is required to extract accurate measurement result for microwave breast cancer detection antennas.This transition is important for avoiding the air gap that happen when we put the conventional antennas that used in breast cancer detection directly on the skin of the patient. An air gap is found between the connector that fed the antenna and the breast tissue, this air gap cause power losses due to transition from antenna to air and then to breast tissue. This power loss make the cancer detection process more difficult either for end-fire or broad side antenna.There are different types of transition published in the last years, one of the most commonly transition was transform from coplanar waveguide (CPW)-to-microstrip line. Zheng, Papapolymerou and Tentezeris [1] had discussed a transition from CPW-tomicrostrip line without vias with afrequency range $(10-40 \mathrm{GHz})$ and this transition used in variety of applications due to its compatibility with RF systems-on-a chip. Zhou and Melde [2] had made a modification and had discussed a transition from coplanar waveguide (CPW)-to-microstrip line with vias to achieve low frequency limit and achieved $36 \mathrm{GHz}$ bandwidth. This transition had been employed on-wafer measurements if microstrip in cases when a low measurement frequency is required. Fang and Wang [3] had discussed a new type of transition from miniaturized coplanar waveguide (CPW) to rectangular waveguide using inductance compensated slot line which almost coveredthe whole X-band (8.2-12.4 GHz) in order to attain the broadband performance and reduce the transition size with 18\%. White, Song and Yason [4] had discussed a transition from a coaxial cable-to-coplanar waveguide (CPW) and had achieved insertion loss less than $0.5 \mathrm{~dB}$ on printed on-glass antenna operate in the range 0.9 to $2.4 \mathrm{GHz}$. Mezzanotte and et al [5] had discussed a FD-TD analysis of coplanar waveguide (CPW) to slotline transitions accounting for air-bridge, shielding effects and coaxial connector as well as interaction between discontinuities. Microwave breast cancer detection had been interest for many years. Microwave breast cancer detection had the potential to detect small tumors because microwave imaging depends on the electrical property distributions in the body [9]. The breast was illuminated with an ultra-wide band pulse and the backscattered signals was recorded [10]. The use of ultra-wide band signal was to provide ultra-wideband reflections or microwave signatures and high resolution [9]. The ultra-wide band signal provide high resolution because the resolution was inversely proportional to bandwidth [10]. The goal was to generate an ultra-wide band frequency. Many researches in this field have discussed various types of antenna working in ultra-wide band range.R. Nilavalan and et al [11] had discussed a wideband microstrip patch antenna designed for breast cancer tumor detection to radiate frequencies in the range 4-9.5 GHz into human breast tissue. Maciej Klemm and et al [12] had discussed a radar-based breast cancer detection using ahemispherical antenna array consists of 16 UWB aperture-coupled stacked-patch antennas. Bourqui and et al [13] had discussed a balanced antipodal vivaldi antenna with dielectric director for microwave breast cancer detection system in the frequency range from 2.4 to 18 GHz.Matteo Bassi and et al [14] had discussed an integrated microwave imaging radar with planar antennas for breast cancer detectionoperates on the broad frequency range from 2 to $16 \mathrm{GHz}$. Mamadou Hady BAHand et al[15] had discussed a vivaldi antenna design for breast cancer imaging within the range of 3.1-10.6 GHz. Malyhe Jalilvandand et al [16] had discussed an ultra-widebandof a hemispherical array of 16 compact bowtie antennas operate in the frequency range of $1.2-7 \mathrm{GHz}$ for breast cancer detection. Most of printed monopole antennas present an ultra-wide band but have a problem in matching 
to a $50 \Omega$ coaxial line, [17]. This paper presents a novel coaxial-to-coplanar waveguide (CPW) that is suitable for breast cancer detection antennas. Ordinarily, the coaxial inner connector's pin is soldered on top of the middle conductor of a Coplanar while the connector's shield is soldered to the ground plane. In this way, the axes of the coaxial and coplanar are perpendicular, presenting a transformation from transverse electromagnetic (TEM) to quasi-TEM.The proposed transition cover the frequency band from 1 to $15 \mathrm{GHz}$. The measurements show good agreement with simulated results. Also, CPW characteristic impedance charts have been developed for different geometries of $\mathrm{CPW}$ and for different relative dielectric constant.This paper is organized as, coplanar waveguide (CPW) design and analysis is described in section II, the proposed structure design and analysis is presented in section III and section IV gives conclusions.

\section{Coplanar Waveguide (Cpw) Design And Analysis}

Coplanar waveguide (CPW), [6] is used for transmission lines where all the conductors are in the same plane; precisely, on the top surface of the dielectric substrate. Coplanar waveguide (CPW) is composed of a median metallic strip separated by two narrow slits from a finite ground plane as shown in Fig.1.

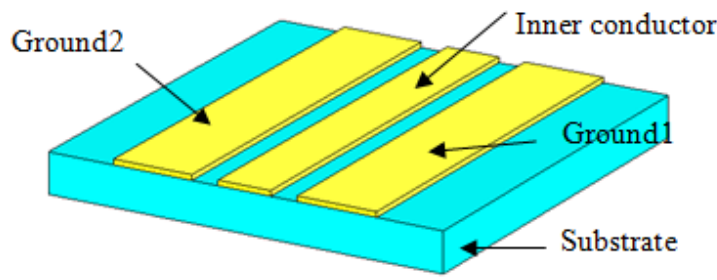

Fig.1. CPW with finite dielectric thickness and finite width ground plane

Coplanar lines advantages arise from the fact that amounting lumped elements is much easier than drilling holes to reach ground plane in other configurations. The performance of coplanar lines is comparable to and sometimes even better than microstrip line in terms of guide wavelength, dispersion, and losses,[6].coplanar waveguides are most promising because of their integration capability with electronic devices and fabrication compatibility with ultra-large scale integration processing (ULSI), [7]. Active elements such as MESFETs can easily be connected to coplanar lines because they are also coplanar in nature. So, coplanar lines are used commonly in monolithic microwave integrated circuits (MMICs), [6]. Borah and Battacharyya [8] use coplanar waveguides to determine the complex permittivity and loss tangent of nano magnetic composite materials over $\mathrm{X}$-band. There is no low-frequency cutoff because of the quasi-TEM mode of propagation. However, the RF electric field between thecenter conducting strip and the ground electrodes tangential to the air dielectric boundary produces a discontinuity in displacement current density at the interface, giving rise to an axial, as well as transverse, component of RF magnetic field, Fig.2.

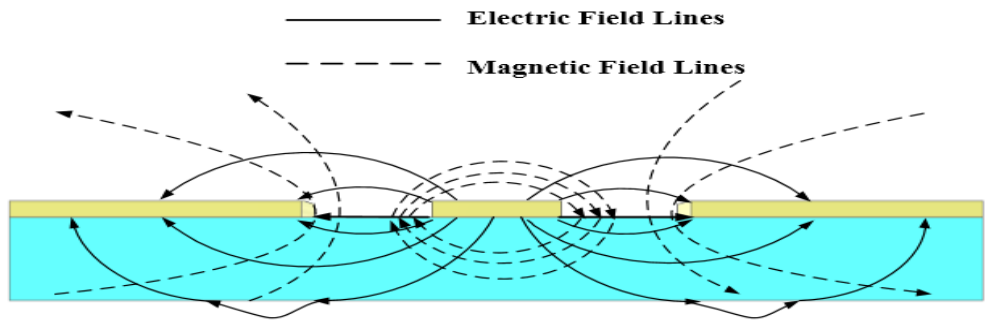

Fig.2. Electric and magnetic field distribution in $\mathrm{CPW}$

There are various types of coplanar waveguides such as CPW with finite dielectric thickness, CPW with finite width ground planes, CPW with a cover shield, conductor backed CPW with a cover shield, conductor-backed $\mathrm{CPW}$, multilayered CPW, asymmetric CPW and asymmetric CPW with finite dielectric thickness.For practical use, CPW with finite dielectric thickness and finite ground plane width is used.

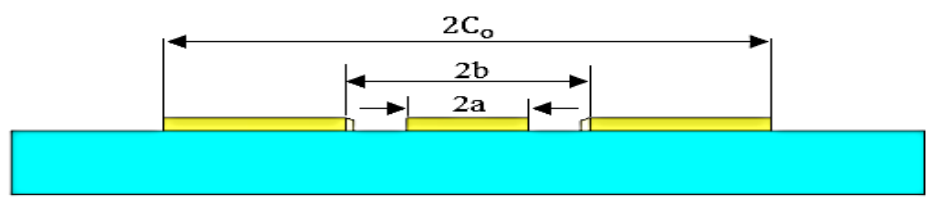

Fig.3. CPW geometry. 
The characteristic impedance of the coplanar waveguide (CPW) transmission line is determined by a, band $\mathrm{C}_{\mathrm{o}}$ as shown in Fig.3. The relative dielectric constant $\epsilon_{\mathrm{r}}$, the effective dielectric constant $\epsilon_{\text {reff }}$, phase velocity $v_{\mathrm{ph}}$, and characteristic impedance $\mathrm{Z}_{\mathrm{ocp}}$, of a transmission line are given as, [5]

$$
\begin{gathered}
\epsilon_{\text {reff }}=1+\frac{\epsilon_{\mathrm{r}}-1}{2} \frac{\mathrm{K}\left(\mathrm{k}_{4}\right)}{\mathrm{K}\left(\mathrm{k}_{4}\right)} \frac{\mathrm{K}\left(\mathrm{k}_{3}\right)}{\mathrm{K}\left(\mathrm{k}_{3}\right)} \\
\mathrm{v}_{\mathrm{ph}}=\frac{\mathrm{c}}{\sqrt{\epsilon_{\text {reff }}}} \\
\mathrm{Z}_{\text {ocp }}=\frac{30 \pi}{\sqrt{\epsilon_{\mathrm{reff}}}} \frac{\mathrm{K}\left(\mathrm{k}_{3}\right)}{\mathrm{K}\left(\mathrm{k}_{3}\right)} \\
\mathrm{k}_{3}=\frac{\mathrm{a}}{\mathrm{b}} \sqrt{\frac{1-\mathrm{b}^{2} / \mathrm{c}_{\mathrm{o}}^{2}}{1-\mathrm{a}^{2} / \mathrm{c}_{\mathrm{o}}^{2}}}
\end{gathered}
$$

$\mathrm{k}_{3}=\sqrt{1-\mathrm{k}_{3}^{2}}$

$$
\begin{gathered}
\frac{\mathrm{K}\left(\mathrm{k}_{3}\right)}{\mathrm{K}\left(\mathrm{k}_{3}\right)}=\frac{1}{\pi} \ln \left\{2\left(1+\sqrt{\mathrm{k}_{3}}\right) /\left(1-\sqrt{\mathrm{k}_{3}}\right)\right\} \\
\text { for } 0.707 \leq \mathrm{k}_{4} \leq 1 \\
\frac{\mathrm{K}\left(\mathrm{k}_{4}\right)}{\mathrm{K}\left(\mathrm{k}_{4}\right)}=\frac{1}{\pi} \ln \left\{2\left(1+\sqrt{\mathrm{k}_{4}}\right) /\left(1-\sqrt{\mathrm{k}_{4}}\right)\right\} \\
\frac{\mathrm{K}(\mathrm{k})}{\mathrm{K}(\mathrm{k})}=\frac{\pi \leq 0.707}{\ln \left\{2\left(1+\sqrt{\mathrm{k}^{2}}\right) /(1-\sqrt{\mathrm{k}})\right\}}
\end{gathered}
$$

Where, $\mathbf{C i s}$ the speed of light in free space and $\mathbf{K}$ is the complete elliptical integral of the first kind, and $\mathbf{K} \square \mathbf{k})=\mathbf{K}\left(\mathbf{k} \square\right.$. A different values of $\mathrm{a}, \mathrm{b}$ and $\mathrm{C}_{\mathrm{o}}$ are considered thencalculate $\mathrm{Z}_{\text {ocp }}$ iscalculated.The process is repeated till the needed value of $\mathrm{Z}_{\text {ocp }}$ is obtained.Figure 4 , shows the flow chart of the CPW impedance calculation tohave the suitable value of $\mathrm{Z}_{\text {ocp }}$ that we need in our design 


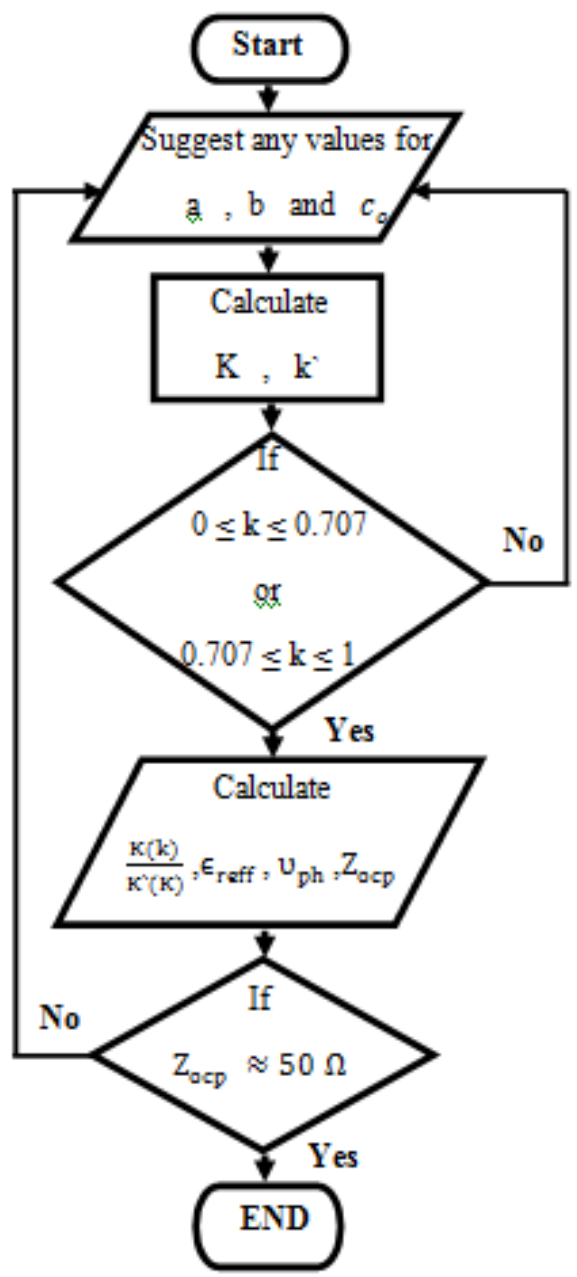

Fig. 4. The flow chart of the CPW impedance calculation

A MATLAB code has been built to calculate the characteristic impedance of CPW in terms of its geometry. The proposed charts give a very fast way in the CPW design process. Figure 5.a shows that the dimensions of a $50 \Omega \mathrm{CPW}$ of $\mathrm{a}=1.0115 \mathrm{~mm}, \mathrm{~b}=1.2775 \mathrm{~mm}$ and $\mathrm{C}_{0}=9.9775 \mathrm{~mm}$ are obtained. One can notice that any other dimensions could be chosen for the $50 \Omega \mathrm{CPW}$. Also, one can notice that effect of the ground plane size on the impedance value, for example for the $50 \Omega \mathrm{CPW}$ with dimension a=1 $\mathrm{mm}$ the impedance value is achieved with $b=1.22 \mathrm{~mm}$ for $C_{0}=9.9775 \mathrm{~mm}$. Also same impedance could be obtained for $b=1.27 \mathrm{~mm}$ and $\mathrm{C}_{0}=7.4775 \mathrm{~mm}$. Also for $\mathrm{b}=1.23 \mathrm{~mm}$ and $\mathrm{C}_{0}=4.977531 \mathrm{~mm}$ same impedance could be obtained, Figs. $5 \mathrm{~b}$ and $\mathrm{c}$. So, the developed charts add another degree of freedom to the designer to obtain same impedance but with different geometries according to the antenna or circuit design situation.

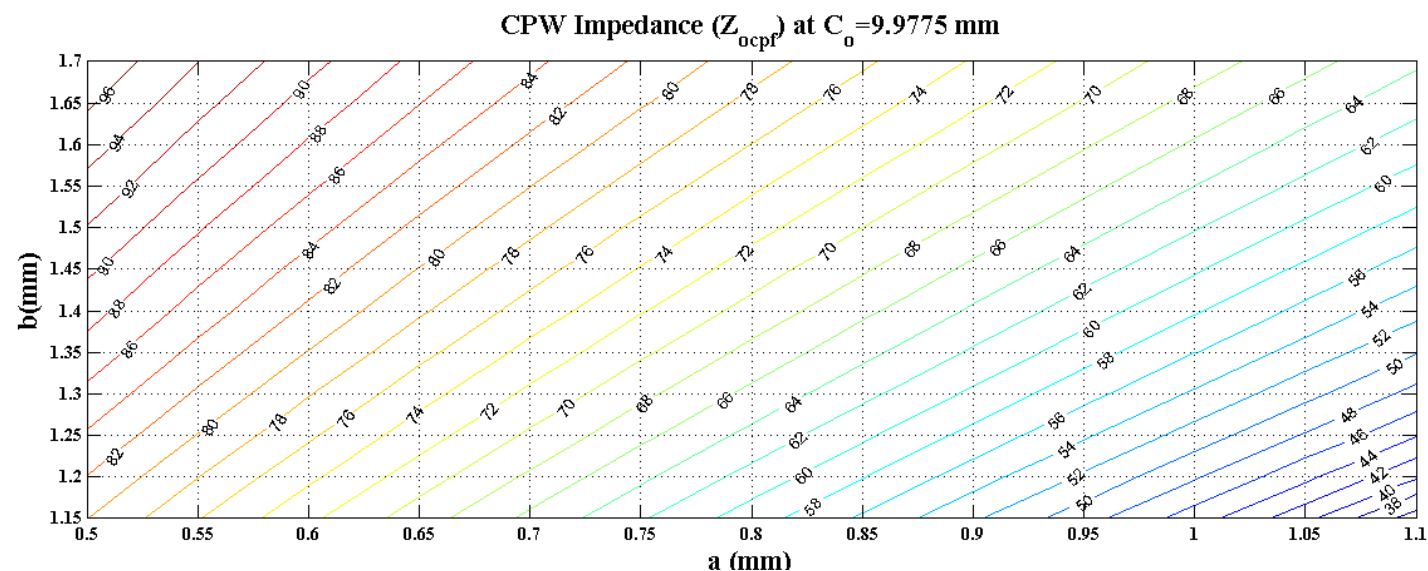

(a) 


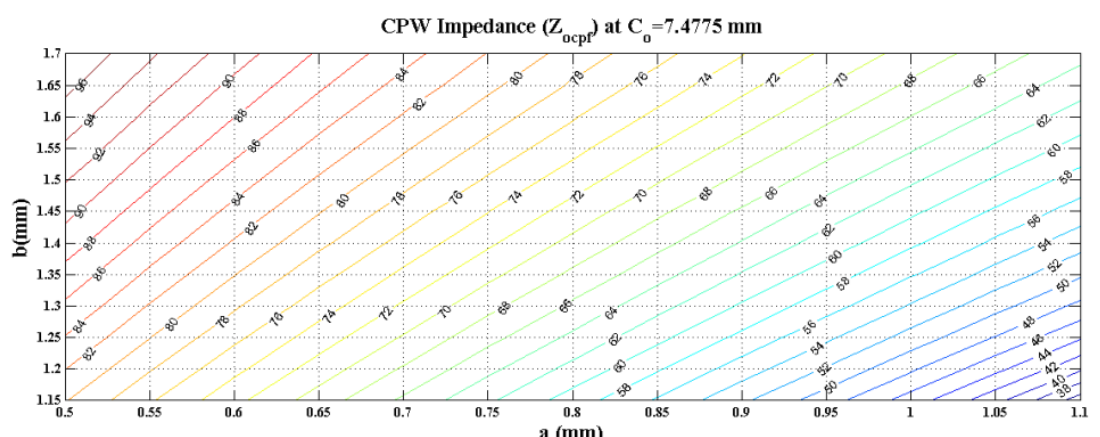

(b)

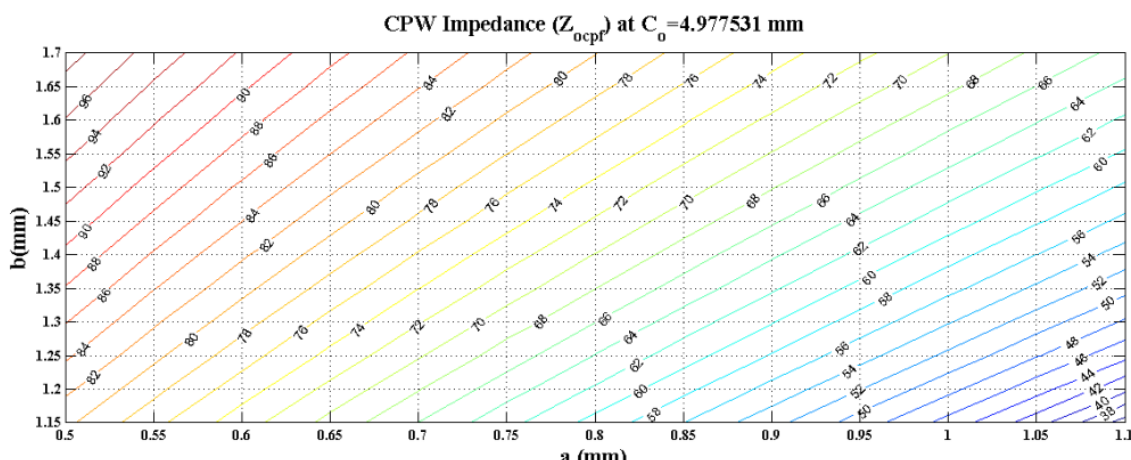

(c)

Fig.5. CPW impedance $\left(Z_{\text {ocpf }}\right)$ charts (a) $\mathrm{C}_{0}=9.9775 \mathrm{~mm}$, (b) $\mathrm{C}_{0}=7.4 .775 \mathrm{~mm},(\mathrm{c}) \mathrm{C}_{0}=4.977531 \mathrm{~mm}$ and $\epsilon_{r}=4.6$.

The previous charts can calculate the dimension of the coplanar waveguide at a specific impedance value. The code is repeated at different substrate materials with different relative dielectric constant. Figure 6 , Fig.7 and Fig.8 show $Z_{\text {ocpf }}$ charts for different values of $\mathrm{C}_{\mathrm{o}}$. Table, 1 show that $50 \Omega \mathrm{CPW}$ different dimensions at $\mathrm{a}=0.95 \mathrm{~mm}$.

Table 1. $50 \Omega \mathrm{CPW}$ dimensions at a $=0.95 \mathrm{~mm}$.

\begin{tabular}{|l|l|l|}
\hline $\mathrm{b}(\mathrm{mm})$ & $\mathbf{c}_{\mathbf{0}}(\mathrm{mm})$ & \multicolumn{1}{|c|}{$\boldsymbol{\epsilon}_{\mathbf{r}}$} \\
\hline $\mathbf{1 . 1 7 1}$ & 9.9775 & 4.6 \\
\hline $\mathbf{1 . 1 6 9}$ & 7.4775 & 4.6 \\
\hline $\mathbf{1 . 1 6 5}$ & 4.977531 & 4.6 \\
\hline $\mathbf{1 . 6 4 5}$ & 9.9775 & 10.2 \\
\hline $\mathbf{1 . 6 4}$ & 7.4775 & 10.2 \\
\hline $\mathbf{1 . 6 2 2}$ & 4.977531 & 10.2 \\
\hline $\mathbf{1 . 2 9 5}$ & 9.9775 & 6.2 \\
\hline $\mathbf{1 . 2 9 3}$ & 7.4775 & 6.2 \\
\hline $\mathbf{1 . 2 8 6}$ & 4.977531 & 6.2 \\
\hline $\mathbf{1 . 0 1 9}$ & 9.9775 & 2.2 \\
\hline $\mathbf{1 . 0 1 8}$ & 7.4775 & 2.2 \\
\hline $\mathbf{1 . 0 1 7}$ & 4.977531 & 2.2 \\
\hline
\end{tabular}

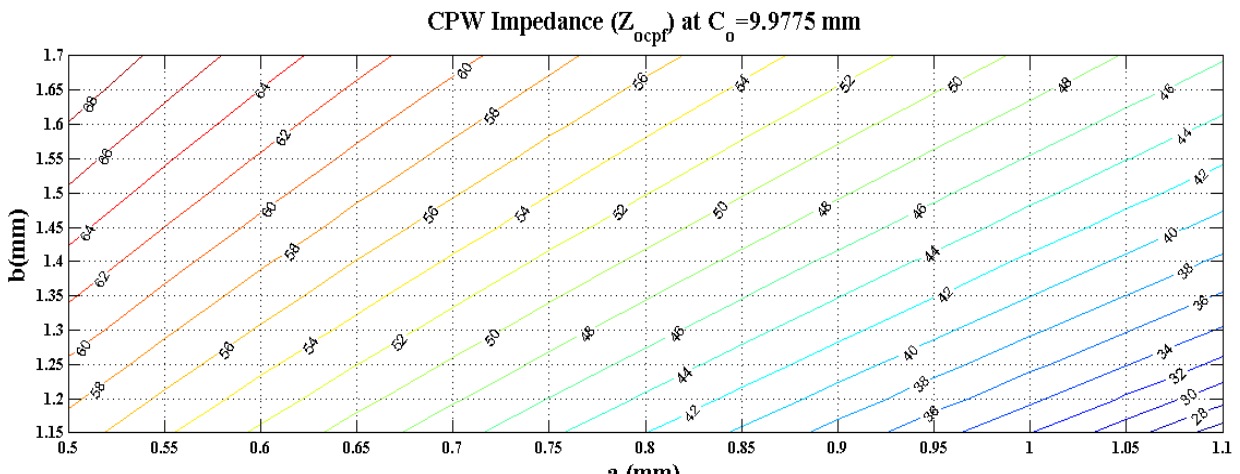

(a) 


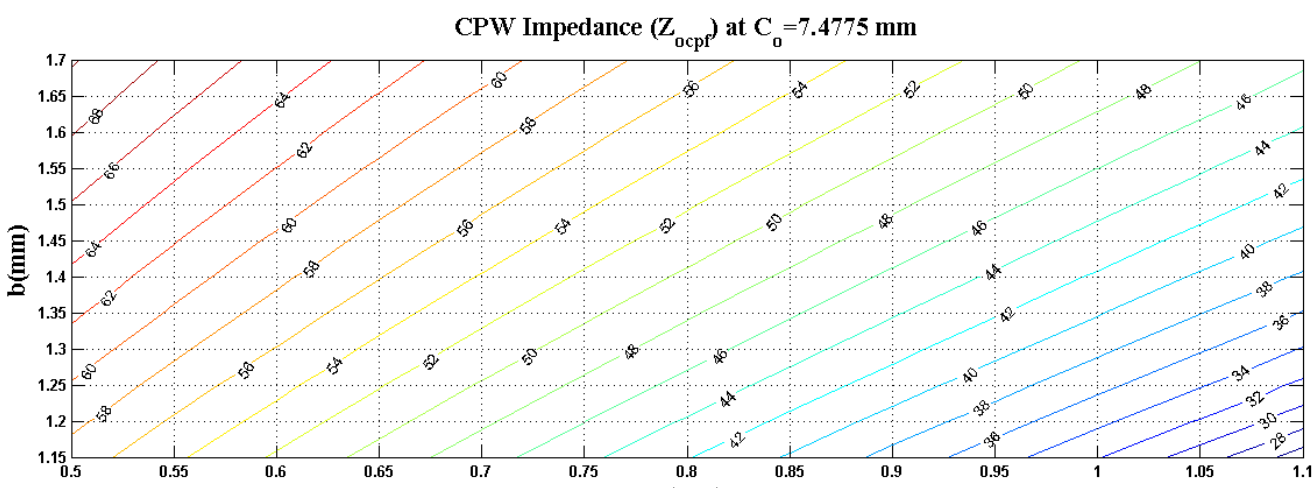

(b)

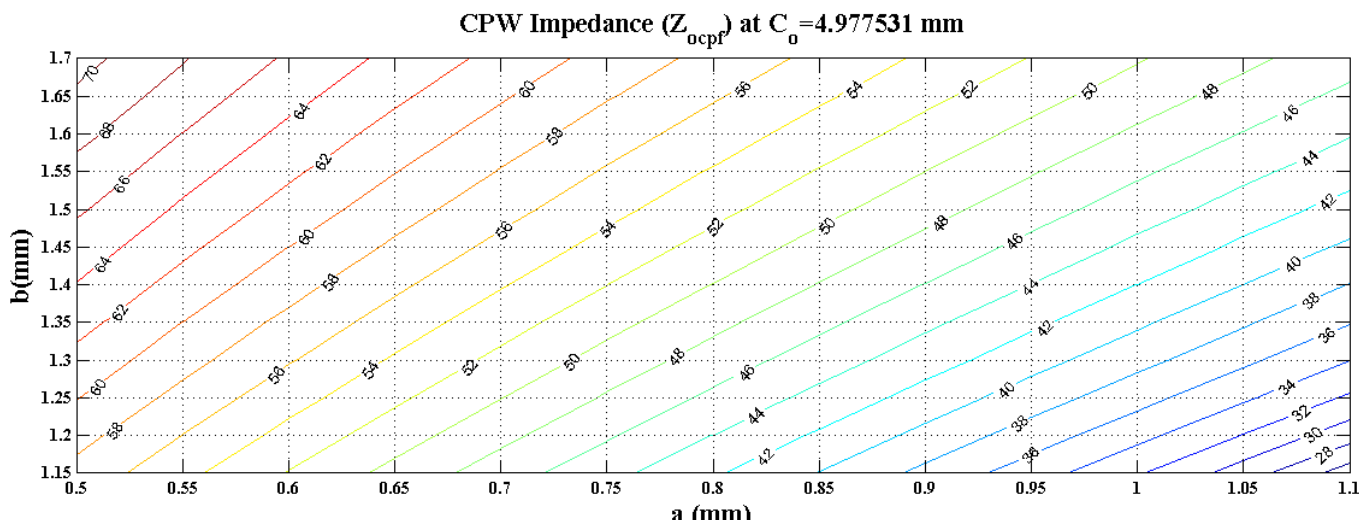

(c)

Fig.6. $\mathrm{CPW}$ impedance $\left(Z_{\text {ocpf }}\right)$ charts (a) $\mathrm{C}_{0}=9.9775 \mathrm{~mm}$, (b) $\mathrm{C}_{0}=7.4775 \mathrm{~mm}$, (c) $\mathrm{C}_{0}=4.977531 \mathrm{~mm}$ and $\epsilon_{r}=10.2$.

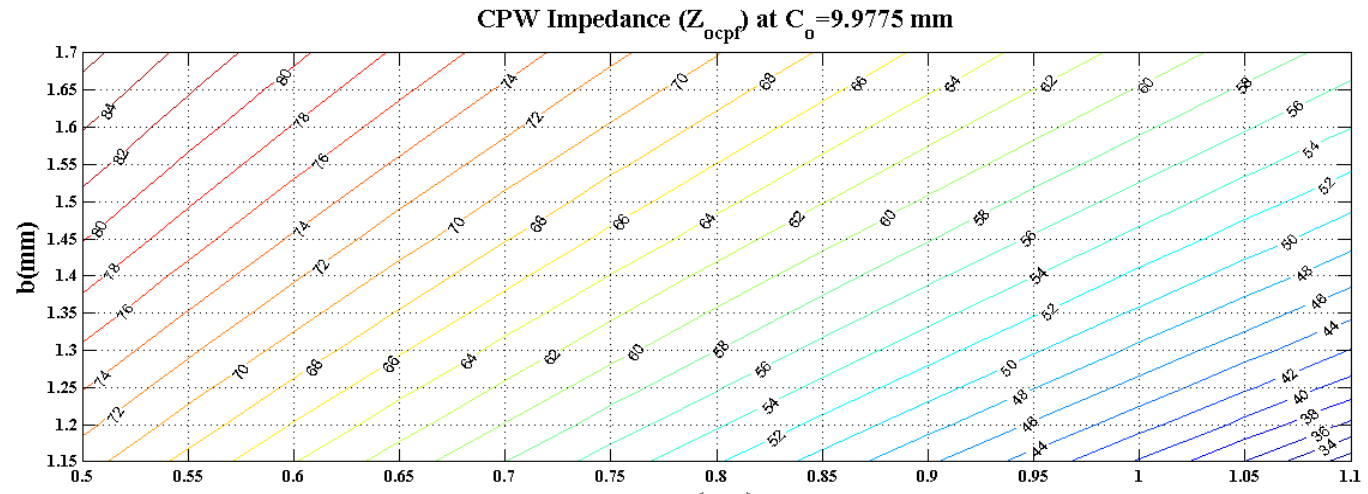

(a)

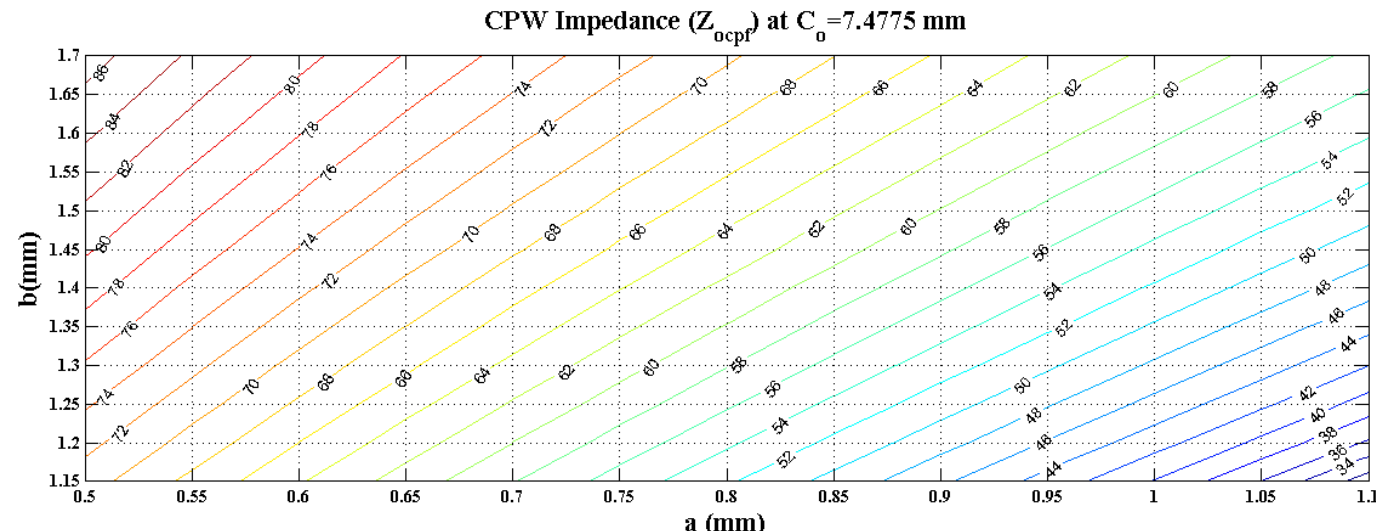

(b) 


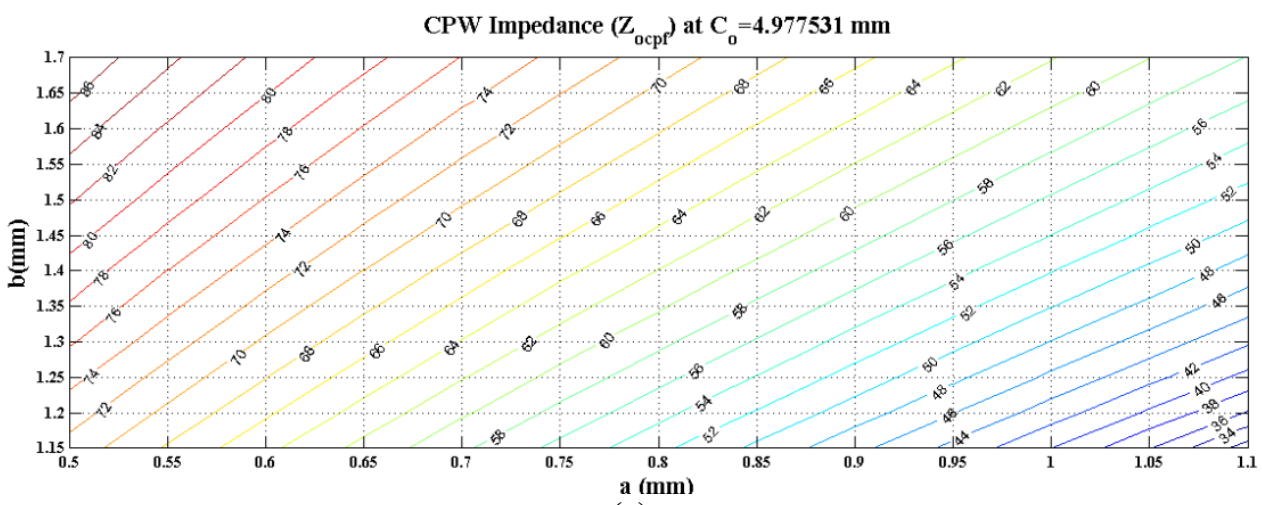

(c)

Fig.7. CPW impedance $\left(Z_{\text {ocpf }}\right)$ charts (a) $\mathrm{C}_{0}=9.9775 \mathrm{~mm}$, (b) $\mathrm{C}_{0}=7.4 .775 \mathrm{~mm}$, (c) $\mathrm{C}_{0}=4.977531 \mathrm{~mm}$ and $\epsilon_{r}=6.2$.

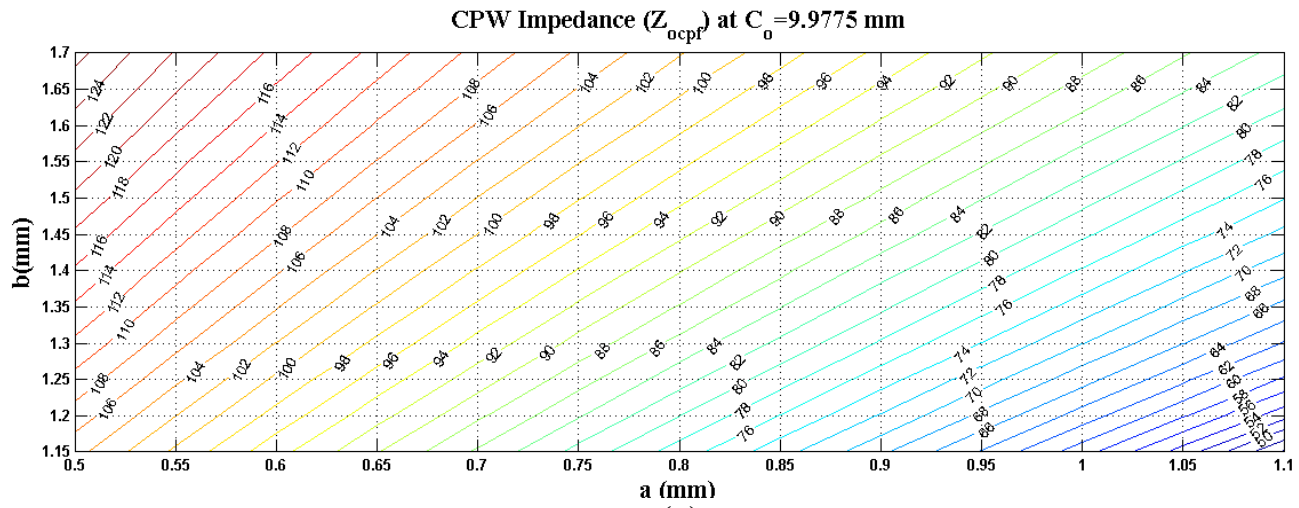

(a)

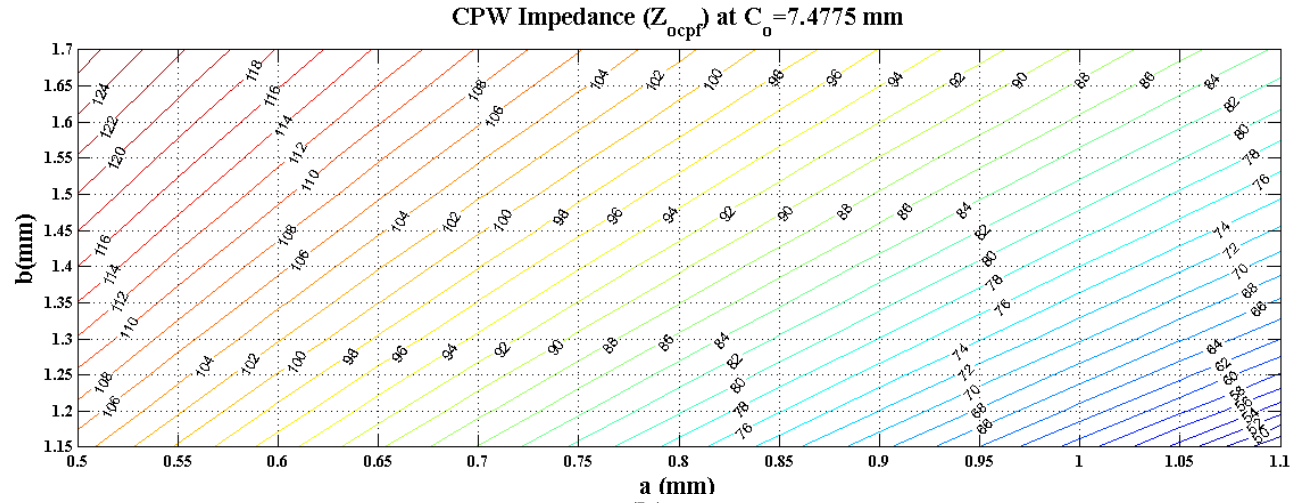

(b)

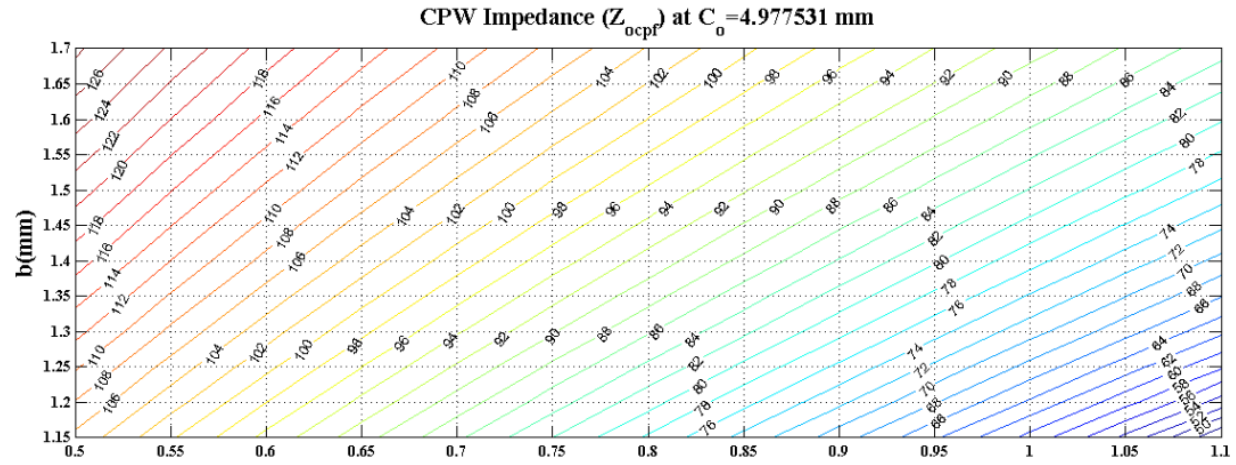

(c)

Fig.8. CPW impedance $\left(Z_{\text {ocp }}\right)$ charts (a) $\mathrm{C}_{0}=9.9775 \mathrm{~mm}$, (b) $\mathrm{C}_{0}=7.4 .775 \mathrm{~mm}$, (c) $\mathrm{C}_{0}=4.977531 \mathrm{~mm}$ and $\epsilon_{r}=2.2$. 


\section{Cpw Proposed Structure, Design And Analysis}

To evaluate this approach, a FR4 substrate with relative permittivity $\epsilon_{r}=4$.6, dielectric thickness of 1.53 $\mathrm{mm}$ and loss tangent of 0.02 is used.A conventional CPW with $\mathrm{a}=1.0115 \mathrm{~mm}, \mathrm{~b}=1.2775 \mathrm{~mm}$ and $\mathrm{C}_{0}=9.9775$ $\mathrm{mm}$ is firstly, considered. A coaxial connector with impedance $50 \Omega$ is connected in the back of the substrate,the coaxial inner connector is connected with the median strip of CPW and the coaxial ground with the outer strips of the CPW, Fig.10.

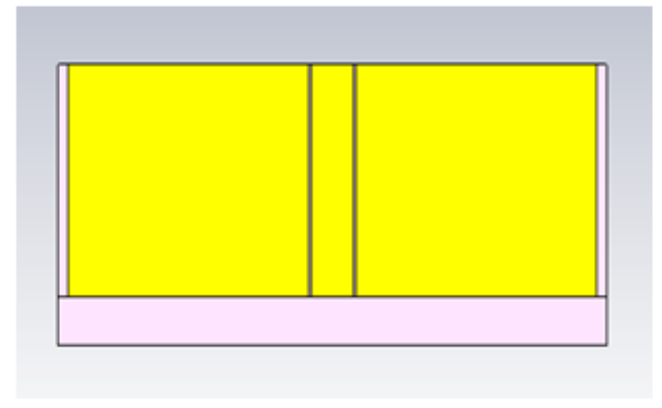

(a)

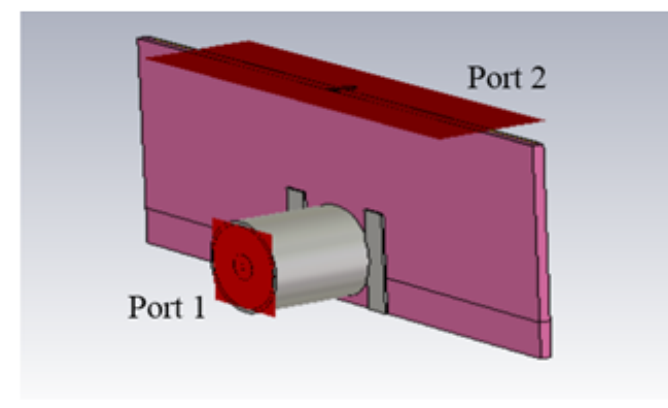

(b)

Fig.9. (a) Conventional CPW front side (b) ConventionalCPW backside

The transformation from coaxial line to CPW is simulated using CST Microwave Studio 2014 software, the S-parameters are shown in Fig.11.

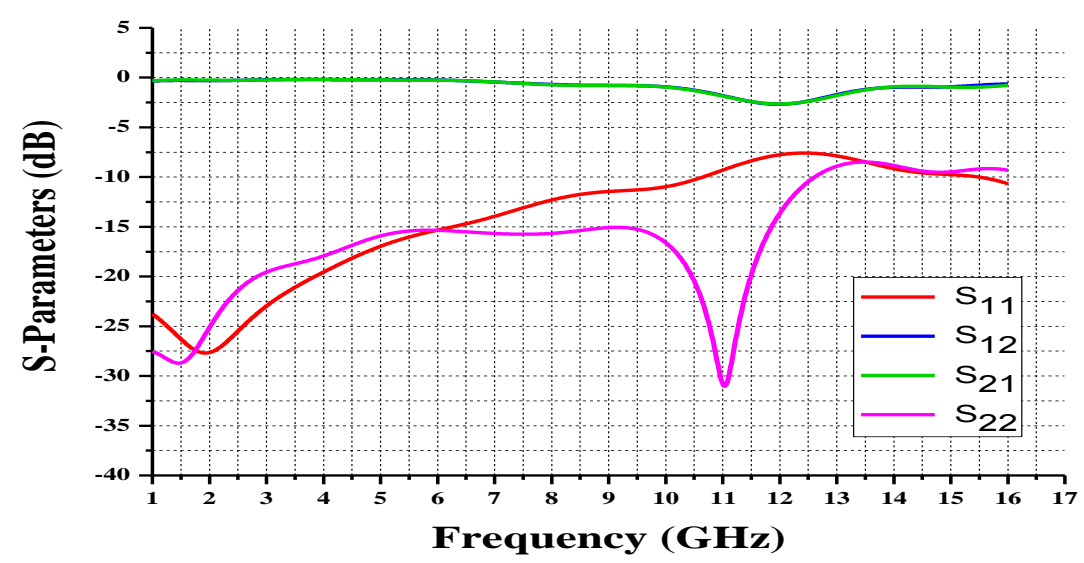

Fig.10. Simulated S-parameters of conventional CPW.

Figure 11 shows that the simulated S-parameters where the operating frequency band extends from 1 $\mathrm{GHz}$ to $10 \mathrm{GHz}$. A slits has been added to the proposed transition for matching purpose [18]. An improvement of about $10 \mathrm{~dB}$ has been obtained. The proposed structure parameter and the parameter values are shown in Fig. 12 and Table 2.

Table 2. CPW parameter values

\begin{tabular}{|l|l|}
\hline A & $2.023 \mathrm{~mm}$ \\
\hline B & $0.266 \mathrm{~mm}$ \\
\hline C & $8.7 \mathrm{~mm}$ \\
\hline D & $6.2 \mathrm{~mm}$ \\
\hline E & $2.7 \mathrm{~mm}$ \\
\hline F & $8.7 \mathrm{~mm}$ \\
\hline G & $3 \mathrm{~mm}$ \\
\hline I & $3.2 \mathrm{~mm}$ \\
\hline J & $1.5 \mathrm{~mm}$ \\
\hline K & $2.5 \mathrm{~mm}$ \\
\hline L & $5 \mathrm{~mm}$ \\
\hline $\mathrm{M}$ & $5.5 \mathrm{~mm}$ \\
\hline
\end{tabular}




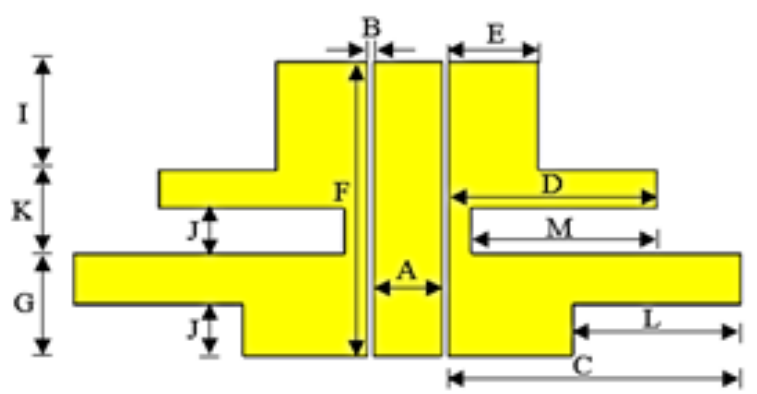

Fig.11.Proposed structure of CPW

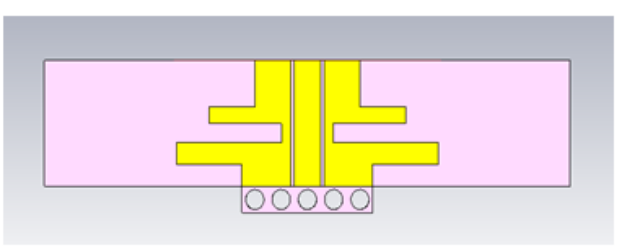

(a)

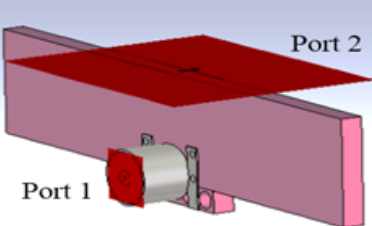

(b)

Fig.12. Simulated proposed CPW (a) front side (b) backside.

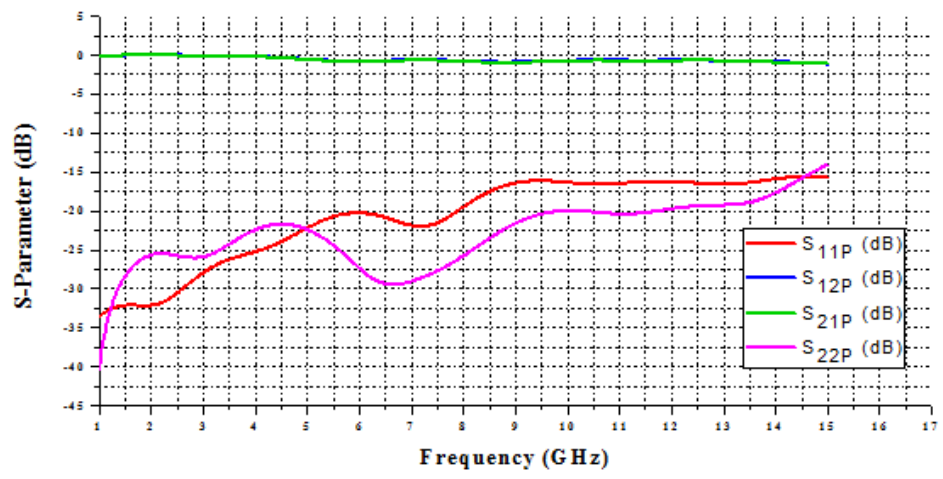

Fig.13. Simulated S-parameter of the proposed transition.

Figure 14 shows that the simulated proposed transition $\mathrm{S}_{21 \mathrm{P}}$ and $\mathrm{S}_{12 \mathrm{P}}$ are almost the same. $\mathrm{S}_{11 \mathrm{P}}$ and $\mathrm{S}_{22 \mathrm{P}}$ are different this due to different feeding schemes one is parallel to the CPW axe and the other is perpendicular to it. Figure 15 show the simulated S-parameters of the conventional CPW and the proposed one. It can be observed, that the return loss $\mathrm{S}_{11 \mathrm{P}}$ of the proposed transition isbetter by $10 \mathrm{~dB}$ than the conventional one $\mathrm{S}_{11 \mathrm{C}}$ and its band extends from $1 \mathrm{GHz}$ to $15 \mathrm{GHz}$.

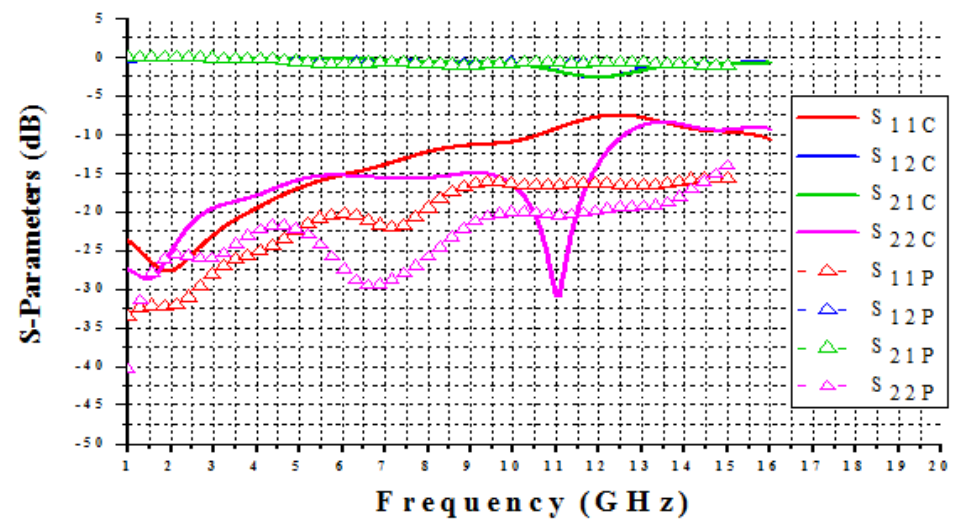

Fig.14. The simulated S-parameters of the conventional and the proposed transition. 
Figures $16 \mathrm{a}$ and $\mathrm{b}$ show the current distributions of the conventional and the proposed transition.

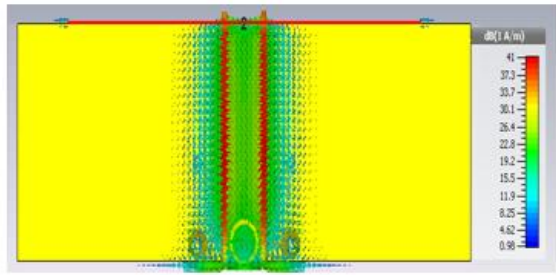

(a)

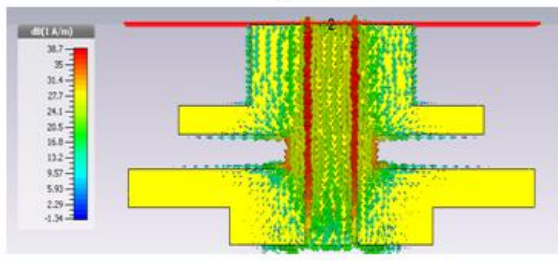

(b)

Fig.15. (a) Current distribution on conventional (b) Current distribution on proposed transition.

Figure 17 shows the fabricated proposed Coaxial-to-CPW transition from front side and from backside. Figure 18 shows the measurement process of the Coaxial-to-CPW transition using Vector Network Analyzer (VNA).

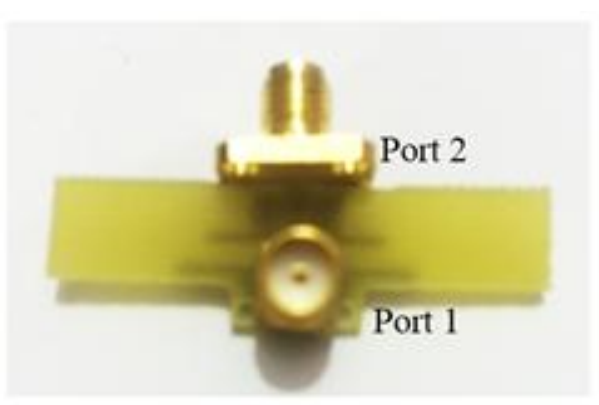

(a)

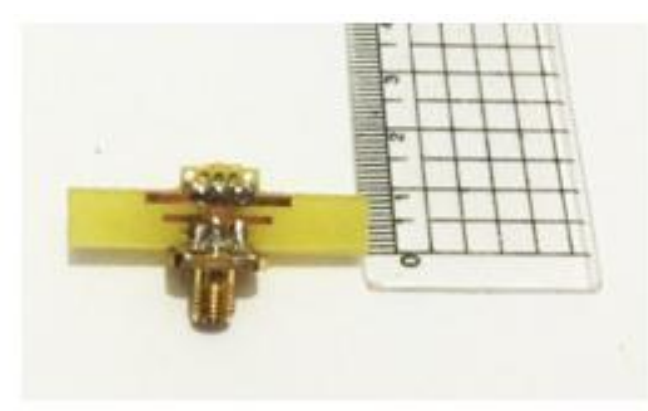

(b)

Fig.16. Fabricated Coaxial-to-CPW transition (a) front side (b) backside.

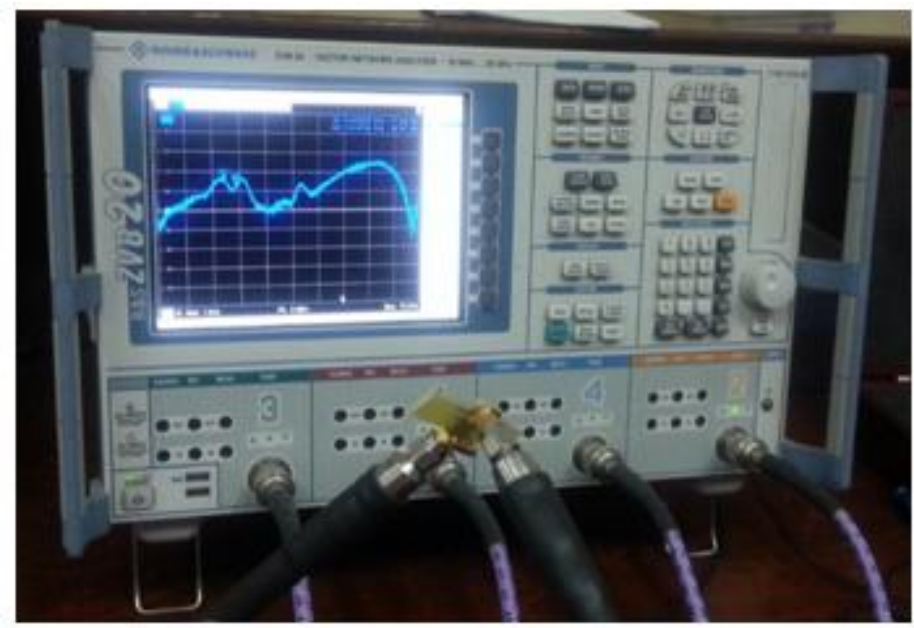

Fig.17.Coaxial-to-CPW transition measurement setup.

Figure 19 shows the measured and simulated return loss and insertion loss of the proposed Coaxial-to$\mathrm{CPW}$ transition. It can be observed, the bandwidth is extended from 1 to $15 \mathrm{GHz}$ which agree well with the simulated results and that's a good result to have a successful transition from Coaxial cable-to-Coplanar waveguide (CPW). 


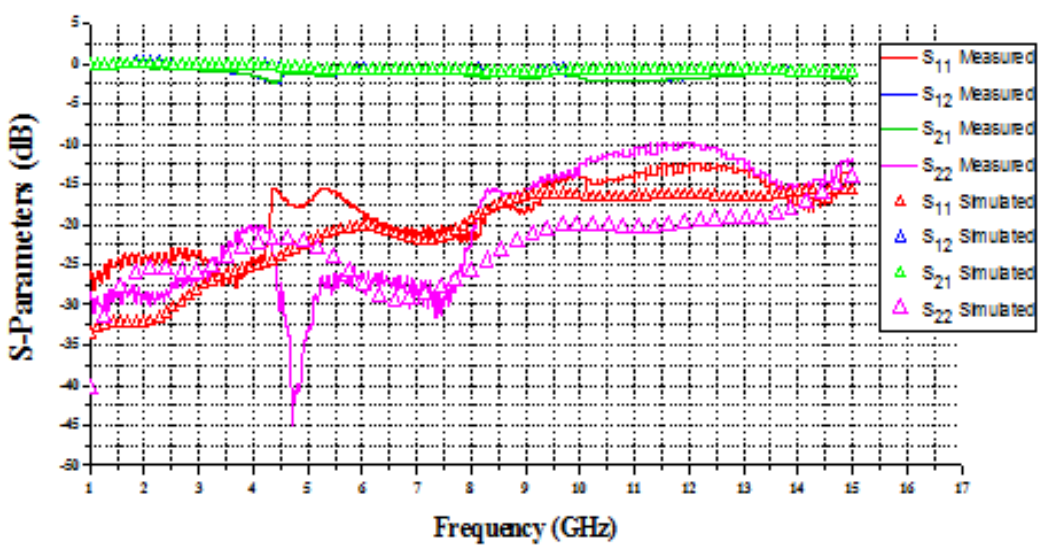

Fig.18. Measured and Simulated Return loss and Insertion loss of Coaxial-to-CPW transition

\section{Conclusion}

A transition from coaxial-to-coplanar waveguide (CPW) for microwave breast cancer detection antennas was presented and design rules based on analytical calculations and simulated one using CST Microwave Studio 2014 simulator were presented. A design charts which add a degree of freedom to the CPW was also proposed. The measured results show that an operating frequency range extends from $1 \mathrm{GHz}$ to $15 \mathrm{GHz}$ compared with the conventional $\mathrm{CPW}$ which extends from $1 \mathrm{GHz}$ to $10 \mathrm{GHz}$ was obtained. Also, an enhancement of about $10 \mathrm{~dB}$ was obtained all over the frequency band. The proposed transition presents a good start to any UWB antenna using a CPW as a feeding scheme.

\section{Refernences}

[1] Guizhen Zheng, J. Papapolymerou, and M. M. Tentzeris, Wideband Coplanar Waveguide RF Probe Pad to Microstrip Transitions without Via Holes, IEEE Microwave and Wireless Components Letters, Volume:13, Year: 2003, Pages:544 - 546

[2] Zhen Zhou, and Kathleen L. Melde, Development of a Broadband Coplanar Waveguide-to-Microstrip Transition with Vias,IEEE Transactions on Advanced Packaging, Volume:31, Year:2008,Pages:861 - 872.

[3] Ruei-Ying Fang, and Chun-Long Wang, Miniaturized Coplanar Waveguide to Rectangular Waveguide Transition Using Inductance-Compensated Slotline,IEEE Transactions on Components, Packaging and Manufacturing Technology, Volume:2,Year:2012,Pages1666 - 1671.

[4] Carson R. White, Hyok J. Song, and Eray Yasan, A Wideband Stick-On Connector for CPW-Fed On-Glass Antennas, ,IEEE Antennas and Wireless Propagation Letters, Volume 9, Year: 2010, Pages: 171 - 174.

[5] P. Mezzanotte, G. Pompei, L. Roselli and R. Sorrentino, FD-TD Analysis of Coplanar Waveguide to Slotline Transitions Accounting for Air-Bridge, Shielding effects and Coaxial Connectors, IEEEMicrowave Conference, 24th European, Volume: 2,Year:1994, Pages: 1929 - 1932

[6] K.C.Gupta, Ramesh Garg, Inder Bahl and Parakash Bahratia,Microstrip Lines and Slot Lines, Second Edition, Boston, London.

[7] B. Nataraj, and K. Porkumaran, CONFORMAL MAPPING ANALYSIS OF VARIOUS COPLANAR WAVEGUIDE STRUCTURES, ICTACT Journal on Communication Technology, Volume: 3, Year:2012, Pages:532-535.

[8] S. Borah, and N. S. Bhattacharyya, Broadband measurement of complex permittivity of composite at microwave frequencies using scalar scattering parameters, Progress In Electromagnetics Research M (PIER M), Volume: 13, Year: 2010, Pages: 53-68.

[9] E. C. Fear, P. M. Meaney, and M. A. Stuchly,Microwaves for breast cancer detection, IEEE Potentials,Volume: 22, Year: 2003, Pages: $12-18$.

[10] E. C. Fear, and M. A. Stuchly,Microwave detection of breast cancer,IEEE Transactions on Microwave Theory and Techniques, Volume: 48, Year: 2000, Pages: $1854-1863$.

[11] R. Nilavalan,I. J. Craddock, A. Preece, J. Leendertz, and R. Benjamin ,Wideband microstrip patch antenna design for breast cancer tumor detection,IET Microwaves, Antennas \& Propagation, Volume: 1,Year: 2007, Pages: 277 - 281.

[12] Maciej Klemm, Ian J. Craddock, Jack A. Leendertz, Alan Preece, and Ralph Benjamin, Radar Based Breast Cancer Detection Using a Hemispherical Antenna Array-Experimental Results, IEEE Transactions on Antennas and Propagation, Volume: 57,Year: 2009, Pages: $1692-1704$.

[13] Jeremie Bourqui,Michal Okoniewski and Elise C. Fear, Balanced Antipodal Vivaldi Antenna With Dielectric Director for NearFieldMicrowave Imaging, IEEE Transactions on Antennas and Propagation,Volume: 58, Year: 2010,Pages: 2318 - 2326.

[14] Matteo Bassi, Michele Caruso, Muhammad Saeed Khan, Andrea Bevilacqua, Antonio-Daniele Capobianco and Andrea Neviani, An Integrated Microwave Imaging Radar With Planar Antennas for Breast Cancer Detection, IEEE Transactions on Microwave Theory and Techniques, Volume: 61, Year: 2013,Pages: $2108-2118$.

[15] Mamadou Hady Bah, Jingsong Hong, Deedar Ali Jamrom, Jia Jun Liang, and Elisee A. Kponou, Vivaldi antenna and breast phantom design for breast cancer imaging,2014 $7^{\text {th }}$ International Conference on Biomedical Engineering and Informatics, Year: 2014,Pages: 90 - 93 .

[16] Malyhe Jalilvand, Xuyang Li, Lukasz Zwirello, and Thomas Zwick, Ultra wideband compact near-field imaging system for breast cancer detection, IET Microwaves, Antennas \& Propagation, Volume: 9, Year: 2015, Pages: 1009 - 1014.

[17] Müzeyyen Karamanoğlu, Mehmet Abbak, Serkan Simșek, A simple and compactCPW-fed UWB printed monopole antenna with defected ground structures, IEEE Electrical and Electronics Engineering (ELECO), 2013 8th International Conference onYear: 2013, Pages: $443-447$.

[18] K. Kumar, and N. Gunasekaran, A Novel Wideband Slotted mm wave Microstrip Patch Antenna, IEEE, Signal Processing, Communication, Computing and Networking Technologies (ICSCCN), 2011 International Conference on Year: 2011, Pages: 1014. 
Author Bibliography

Asmaa Fereg Desoukey is a Post Graduate Student (master), Alexandria University, Egypt. She was born in Kafer El-Shiekh, Egypt on August, 1989. She is a demonstrator at Higher Institute of Engineering and Technology (HIET) in Kafer El-Shiekh, Egypt.

Tamer ABOUELNAGA was born in 1976. He received his M.Sc. and his Ph.D. in 2007 and 2012 from Ain

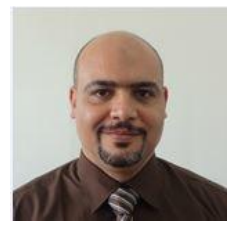

Shams University respectively. His research interests include antennas, microstrip circuit components and RFID system components.

Mazhar B. Tayel was born in Alexandria, Egypt on Nov. 20th, 1939. He was graduated from Alexandria

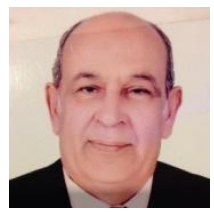
University Faculty of Engineering Electrical and Electronics department class 1963. He published many papers and books in electronics, biomedical, and measurements. Prof. Dr. Mazhar Basyouni Tayel had his B.Sc. with honor degree in 1963, and then he had his Ph.D. Electro-physics degree in 1970. He had this Prof. Degree of elect. And communication and Biomedical Engineering and systems in 1980. Now he is Emeritus Professor since 1999. From 1987 to 1991 he worked as a chairman, communication engineering section, EED BAULebanon and from 1991 to 1995 he worked as Chairman, Communication Engineering Section, EED Alexandria. University, Alexandria Egypt, and from 1995 to 1996 he worked as a chairman, EED, Faculty of Engineering, BAU-Lebanon, and from 1996 to 1997 he worked as the dean, Faculty of Engineering, BAU Lebanon, and from 1999 to 2009 he worked as a senior prof., Faculty of Engineering, Alexandria. University, Alexandria Egypt, finally from 2009 to now he worked as Emeritus Professor, Faculty of Engineering, Alexandria University, Alexandria Egypt. Prof. Dr. Tayel worked as a general consultant in many companies and factories also he is Member in supreme consul of Egypt. E.Prof. Mazhar Basyouni Tayel. 